\title{
Construction Industry Perspective on Planning and Scheduling Content
}

\author{
Saeed Rokooei ${ }^{1}$, Neil Pickavance ${ }^{2}$, Alireza Shojaei ${ }^{3}$ and Andrew Ross ${ }^{4}$ \\ 1 Mississippi State University, Starkville, USA, srokooei@caad.msstate.edu \\ 2 Liverpool John Moores University, Liverpool, UK, N.M.Pickavance@2017.ljmu.ac.uk \\ 3 Mississippi State University, Starkville, USA, shojaei@caad.msstate.edu \\ 4 Liverpool John Moores University, Liverpool, UK, A.D.Ross@ljmu.ac.uk
}

\begin{abstract}
This paper succinctly reports a study performed to explore planning and scheduling as the two key components of every project. Construction project managers devote a major portion of their daily time to develop, revise, and update their schedules. The same emphasis exists in construction education where the content is provided in one or two major courses through which students learn the basics and practice to develop project schedules with a high level of accuracy. To get inputs from industry and use them in the scheduling training process, a quantitative research method was utilized in the summer and fall 2019. The results indicated that scheduling as a major division has a strong presence in companies with different sizes, number of projects, and work experience. The results also indicated that different subcontractors have different levels of knowledge and competency in knowing and using time management tools. In addition, the results emphasized the importance of traditional time management techniques and methods. Better coordination between entities, more scheduling training for all, and team support of planners are reported as effective ways to improve the scheduling level. The outcomes help construction educators to revise their training material to correspond to the construction industry's needs.
\end{abstract}

(c) 2020 The Authors. Published by Budapest University of Technology and Economics \& Diamond Congress Ltd Peer-review under responsibility of the Scientific Committee of the Creative Construction Conference 2020.

Keywords: construction, education, planning, scheduling, time management

\section{Introduction}

Modern construction management makes extensive use of traditional scheduling for planning and control of projects. Gantt charts, critical path method, and short-term schedules still appear to be the preferred choice of contractors in the US and UK. However, recent years have seen increasing suspicion that traditional scheduling may have limitations in its ability to accurately forecast long-terms plans. Industry advocates of social-based collaborative planning such as Last Planner System ${ }^{\mathrm{TM}}$, Agile Project Management, and Lean Construction, share a view that traditional construction scheduling is too rigid and deterministic to provide long-term forecasting across project lifecycles. Some believe the prevalence of delay and disruption is often related to the planning and scheduling function, with little regard for the caliber of operational execution behind the program strategy. Despite this, traditional construction scheduling is still mostly specified by clients and used by contractors in the construction industry today.

\section{Literature review}

In construction, the Critical Path Method of project planning and scheduling remains the most commonly used technique in project environments [1], despite newer methods such as Lean Construction, Last Planner ${ }^{\circledR}$ System, and Agile Project Management being promoted and made available to industry practitioners. However, if recognizing the 1950 s as a general start point of modern project management via 
the development and adoption of logic network analysis the planning, management, and control of construction projects was traditionally presented in Gantt chart format with critical trades identified. Originating from post-war industrial, military, and production environments the transposition of critical path method (CPM) scheduling technique into the construction and allied industries was recorded in " $\mathrm{A}$ non-computer approach to the critical path method for construction" [2]. With similarities to Program Evaluation and Review Technique (PERT), CPM is based on mathematical and algorithmic models of logic networks applied to production scheduling [3]. Construction activities, key milestones, and critical trades are sequentially mapped and are used to forecast the project planned intent, with this deterministic approach remaining a significant method of planning and project control in modern construction management [4]. Government-commissioned construction reports are intentioned to provide best-practice strategies on key issues of the time. Influenced by wider economic pressures many focus on project and program delivery improvements through partnering and collaborative working [5], program performance and efficiency [6], reports to check and measure the success of previous reports [7] to force a generationallevel shift in cost and program production improvements [8]. While demanding responses from industry on productivity problems Government reports can offer little insight into the granular reasons behind program and production limitations in industry. Official codes of practice provide established written frameworks for project management and delivery, however these lean towards being largely procedural in nature [9]. The Project Management Body of Knowledge [10] similarly fails to address the complex nature of construction planning and scheduling in complex and fragmented contracting environments. The Code of Practice for Program Management in the Built Environment [11], despite being an authoritative manual, poorly interprets the practical application of the planning role in contracting environments and sets out guidance similar to construction texts. Project planning can face a challenge in organizations where projects are strongly commercially led, often due to a culture of weak project management and leadership. The responsibility for undertaking the planning and scheduling role in construction can vary from a dedicated planner / scheduler, or a project manager, or construction manager. Depending on project size and company culture there may be no formal allocation of the project planning role. This loose definition, weak support, and poor recognition of the role led [12] to question what project planners do. Similar direct querying on whether construction project planning was doing its job [13] demonstrates some misunderstanding of the nature of the execution of the planning role and the responsibilities of the wider team in supporting the project planning function. Poor attitudes towards a strong and centralized planning entity has seen a creeping disdain and the search for alternative planning methodologies. Imported from automotive and production industries from the 1990s onwards alternatives to traditional planning and scheduling, and broadly categorized as collaborative planning, such modern methods as Lean Construction [14] and Last Planner ${ }^{\circledR}$ System [14] are purported to provide a trust-based collaborative alternative to the rational-technical nature of critical path method planning. Other modern methods aimed at supplementing or in many instances replacing traditional planning and scheduling are Agile Project Management, AgiLean Project Management, Six Sigma, and Lean Six Sigma. While characterized by continuous improvement processes, reduction of waste, collaborative working, greater social trust in project teams, greater inclusivity of site work-crews, and work schedule agility these methods have not established themselves as successors to the traditional construction planning and scheduling methods that are still first-choice of employers and contractors in the construction industry.

\section{Methodology}

The main purpose of this study was to explore the status of scheduling tools and approaches used in industry. This study was a joint effort taken by two research universities in the U.S. and U.K. to investigate the similarities and differences between the construction companies in these two countries. To reach this study's objective, the following research questions formed the research approach:

- What scheduling tools, methods, and methods does the construction industry utilize?

- How do scheduling professionals perceive subject content taught in construction programs?

- What are the similarities and differences between construction companies in the U.S. and U.K? 
Construction scheduling is a core course in construction programs, which is being taught consistently across various schools; however, the notion of scheduling in different companies is not perceived in a similar way. One main reason for this situation is the variety of scheduling tools, techniques, and methods that are employed by construction companies. Knowing these two points helps construction educators to adjust their course content and the tools and methods they use to convey the scheduling concept and its application to their students. In a broader scope, it would be even more helpful to know the best practices in the U.S. and compare it with another country. For brevity, this paper strives to only answer the first two questions, and the third question is discussed in another publication. Two research groups from Mississippi State University - U.S. and Liverpool John Moores University - U.K. joined in Spring 2019 to discuss problem statement, research objectives, methodology, and administration. After a preliminary series of discussions, a timeline was provided, and a quantitative research method was chosen to conduct the study. A survey was designed to cover the main tools, methods, and approaches used in scheduling. The survey was designed in four sections. Section one included the demographic and general information of respondents. The second section explored the awareness and competence of various entities involved in construction projects. The third section examined the importance and use of different time management methods and tools. And finally, in section four, factors to improve the outcome of project time management and scheduling were investigated in the fourth section. The survey was sent to different construction companies who serve as an industrial advisory board member at Mississippi State University and as a result, thirtyfour responses were received. The gathered data were compiled and modeled in a statistical software. Through the first round of data modeling, a few outliers were identified, and some data imputations were utilized.

\section{Results}

Survey responses were collected within a month from different companies. Except for one respondent, all other participants fully responded to the survey where $91 \%$ of them were male. As shown in Table 1, the frequency of all age groups is almost evenly distributed.

Table 1. Percentage of each age group

\begin{tabular}{ccccccc}
\hline Age Group & Under 26 & $26-35$ & $36-45$ & $46-55$ & $56+$ & Total \\
\hline Percentage & 9 & 28 & 21 & 18 & 24 & 100 \\
\hline
\end{tabular}

The majority of participants (88\%) were working in GC companies and the rest were working in trade/subcontractor companies. The reported work experience of participants is shown in Figure 1. The results also showed that $53 \%$ of participants reported their positions as project manager, $38 \%$ as scheduler, and $9 \%$ of participants had other positions. The size of companies that participants worked in is specified in Table 2. As shown, the majority of sample group were involved in medium and large size companies.

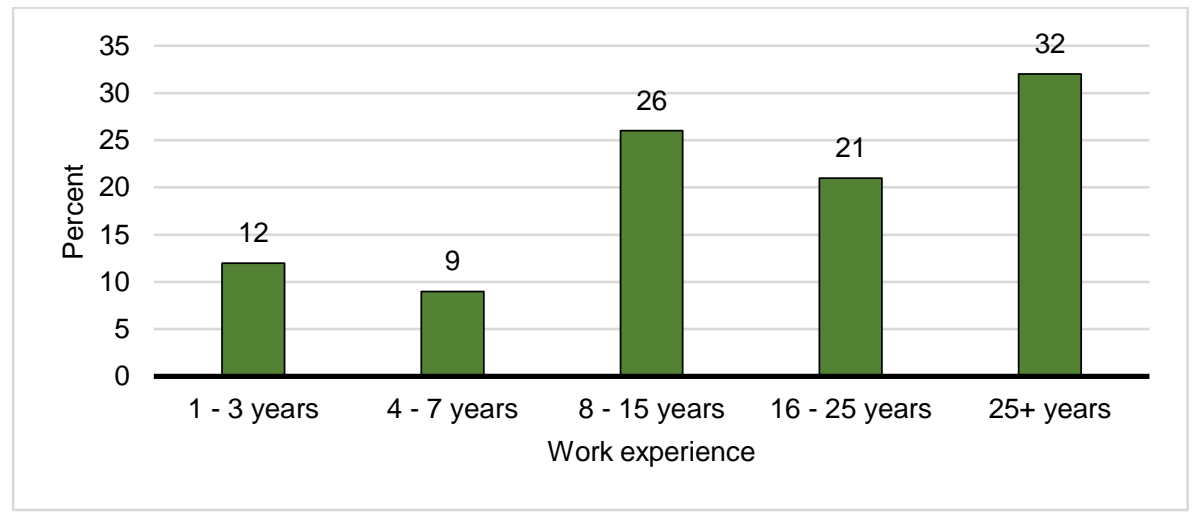

Fig. 1. Frequency of work experience groups 
Table 2. Percentage of each size of company

\begin{tabular}{ccccccc}
\hline Size of Company & 10 or less & $11-50$ & $51-200$ & $201-500$ & $501+$ & Total \\
\hline Percentage & $3 \%$ & $12 \%$ & $32 \%$ & $3 \%$ & $50 \%$ & 100
\end{tabular}

Participants also specified how often they update schedule. Possible responses were "No update", "Weekly or less", "Biweekly", "Monthly", and "Every 3 months", and the percentages were 3\%, 9\%, 56\%, 32\%, and 0\%, respectively. This indicates that the majority of schedules are updated every two weeks. Moreover, participants were asked to rate what percentage of their projects are completed based on the initial duration. The provided percentages were $10 \%$ or less, $11-20 \%, 21-40 \%, 41-60 \%$, and $61+\%$. The percentage of each category is reported as $0 \%, 9 \%, 9 \%, 15 \%$, and $68 \%$, respectively. In the next section, participants were asked to specify the main use of their schedules. For this question, participants were allowed to select only one item. Figure 2 shows the percentage of each possible use of the schedule based on the participants' report.

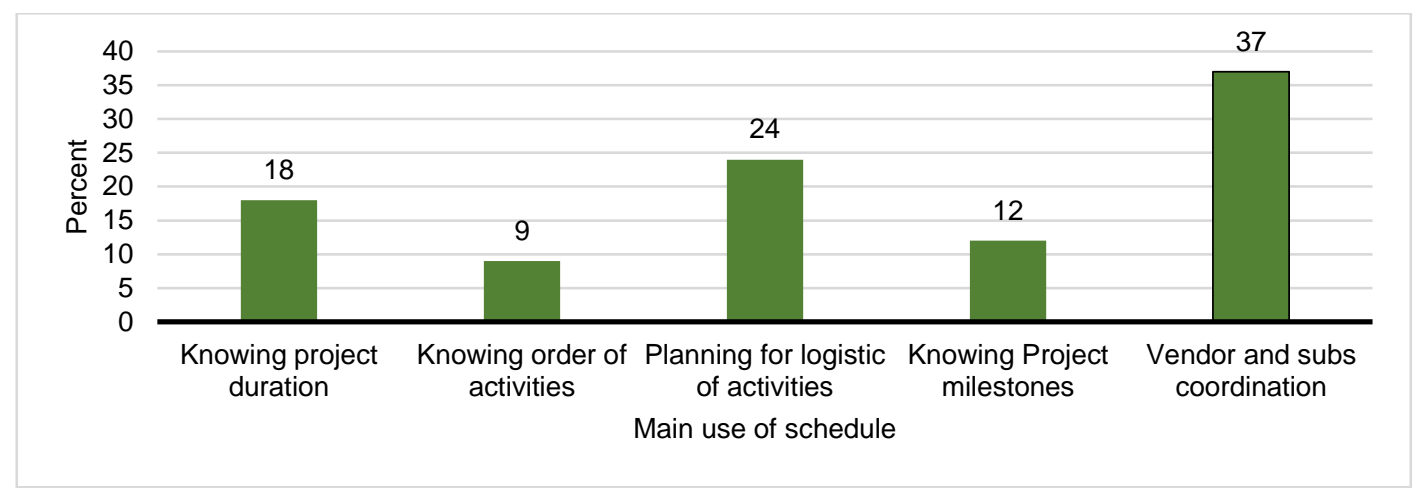

Fig. 2. Percentage of possible uses of schedule

Participants also reported the main issue with Critical Path Method (CPM) in their project scheduling, Table 3 shows the percentage of possible issues, reported by participants.

Table 3. Percentage of each possible issue derived from using CPM

\begin{tabular}{ccccccc}
\hline Issue & Inaccuracy & Complexity & $\begin{array}{c}\text { Time } \\
\text { consumption }\end{array}$ & Too much info & $\begin{array}{c}\text { Different } \\
\text { software }\end{array}$ & Total \\
\hline Percentage & $24 \%$ & $29 \%$ & $35 \%$ & $6 \%$ & $6 \%$ & 100 \\
\hline
\end{tabular}

Additionally, participants were asked to specify the importance/usage of following time management methods/tools using a 5-level Likert scale from 1: Very Low to 5: Very High. Figure 3 illustrates the average score of each tool/method out of 5. 


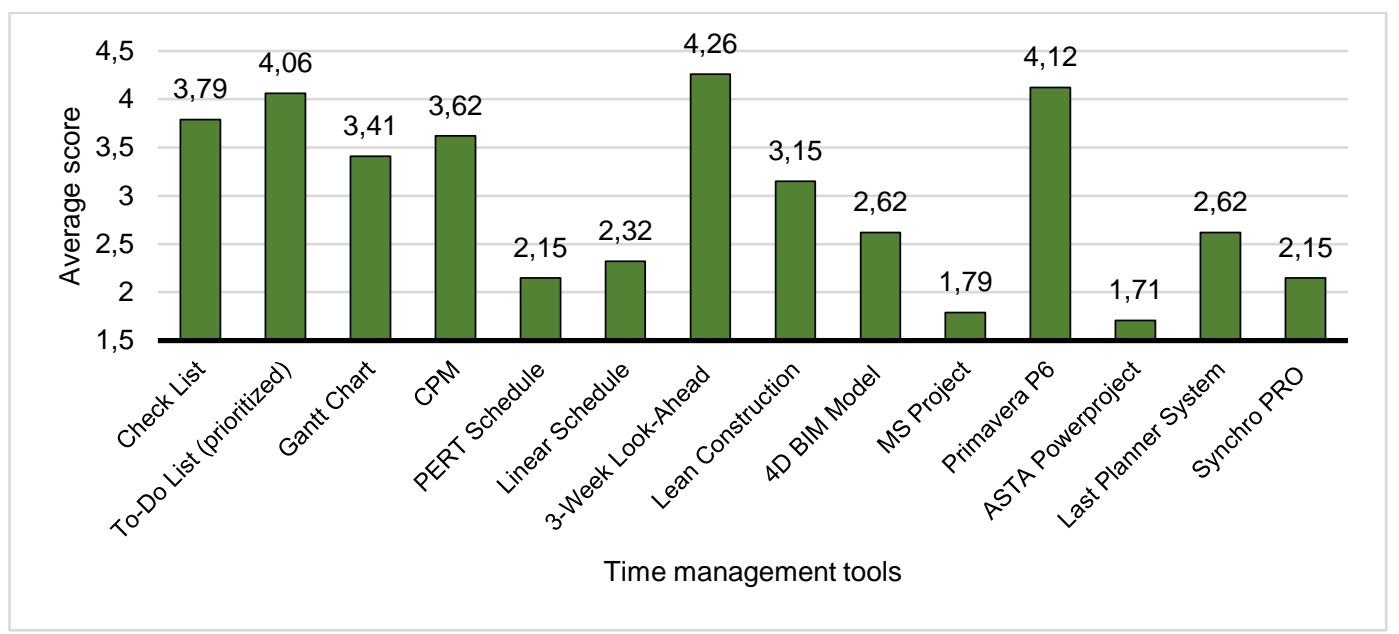

Fig. 3. Average score of time management tools

Finally, participants were asked to rate the importance of possible factors to improve the outcome of project time management/scheduling. The relative average weight of each improvement factor is shown in Table 4 in which "Better coordination between entities" with a considerable difference with other factors has gained the highest score.

Table 4. Relative average weight of each improvement factor (out of 5)

\begin{tabular}{lc}
\hline Improvement Factor & Relative Average Weight \\
\hline Better coordination between entities & 4.47 \\
Using more experienced people in scheduling & 4.12 \\
More Scheduling training for all & 4.09 \\
Team support of Planner/ Scheduler & 4.06 \\
Using advanced technology & 3.79 \\
Better Program Schedule Ownership (Planner or Project Manager) & 3.76 \\
Earlier Development of Construction Program & 3.71 \\
Quality of Subcontractor Generated Program & 3.68 \\
More frequent schedule updates & 3.56 \\
Simplifying the schedules & 3.53 \\
Accurate Resourcing of Program & 3.21 \\
Formal Stages of Program Development (Gateways) & 2.97 \\
Putting rewards and penalties for time deviations & 2.79 \\
\hline
\end{tabular}

\section{Discussion}

Planning and scheduling are a core component of construction curricula, which are typically offered in one course at junior or senior level. Unlike many other construction subjects, the content of scheduling courses has been relatively unchanged for a long time in academia. However, the construction industry constantly strives to improve planning and scheduling methods to attain higher quality, shorter durations and lower costs. These two situations necessitate a bridge to connect these two sectors and bring both to the same pace and direction. One major input for such modification is industry professionals' feedback on common scheduling tools and methods used in construction programs. The current study aims to provide input for construction educators and administers in planning and scheduling courses. One noticeable point is the frequency of age and work experience of participants. The results show that those who are involved or in charge of scheduling are of different age groups and with different work experiences. This balanced mixture can make scheduling an agile sector of the construction companies. However, the meaningful difference between items categorized based on the time spent for scheduling tasks indicates two important points: a high level of involvement (i.e. developer) or a low level of interaction with scheduling tasks (i.e. coordinator). Another important point derived from the response is the relatively low score of new scheduling 
tools/methods used in companies. For example, 4D BIM, ASTA, and Synchro have been scored considerably lower than traditional check list, to-do list, and Gantt chart. Finally, as indicated by the results, the main improvement approach is a more effective coordination. For construction students preparing to enter this area, this translates to a better communication, working in multidisciplinary teams, and having holistic perspectives.

\section{Conclusion}

Scheduling is a vital task in construction project management. Cost and time are two major drivers for every project which can be properly managed through an accurate schedule. Knowing how professionals think about the different aspects of the scheduling process, tools and methods, and best practices helps construction educators to provide students with the course content accordingly. This study was designed to obtain the construction perspective on the scheduling area and exhibit the trends, similarities, and differences between various construction entities. The results indicated that complexity and time consumption are the two main obstacles in using scheduling techniques and methods. Construction students should learn how to efficiently develop a schedule and incrementally revise it. Another noticeable point is the importance of simple and traditional time management tools. Although new advanced scheduling tools, techniques, and methods seem intriguing and can absorb students' attention, it should be noted that construction graduates should be competent in developing and using the common - and sometimes outdated - tools to communicate with other individuals, especially their seniors. Finally, it is important to note the relatively level of familiarity with time management tools in trades/ subcontractors. Knowing the current situation enables construction graduates to more effectively communicate with this group. Although the results highlight different aspects of scheduling in the construction industry through the lens of professionals, this paper does not generalize the outputs due to the sample size. Having a larger sample size warrants more reliable results. The next phase of this study will include more companies across the nation with various expertise.

\section{References}

[1] Ammar, M. A. (2012). LOB and CPM Integrated Method for Scheduling Repetitive Projects. Journal of Construction Engineering and Management, 139(1), 44-50. https://doi.org/10.1061/(asce)co.1943-7862.0000569

[2] Fondahl, J. W. (1962). A Non-computer Approach to the Critical Path Method for the Construction Industry.

[3] Aquilano, N. J., \& Smith, D. E. (1980). A Formal Set of Algorithms for Project Scheduling with Critical Path Scheduling/Material Requirements Planning. Journal of Operations Management, 1(2), 57-67. https://doi.org/10.1016/0272-6963(80)90013-3

[4] Agyei, W. (2015). Project Planning and Scheduling Using PERT and CPM Techniques with Linear Programming: Case Study. International Journal of Scientific \& Technology Research, 4(8), 222-227.

[5] Latham, M. (1994). Constructing the Team: Joint Review of Procurement and Contractual Arrangements in the UK Construction Industry. UK: Department of the Environment.

[6] Egan, J. (1998). Rethinking Construction: Report of the Construction Task Force on the Scope for Improving the Quality and Efficiency of UK Construction. London: Department of the Environment, Transport and the Regions.

[7] Wolstenholme, A., Austin, S., Bairstow, M., Blumenthal, A., Lorimer, J., McGuckin, S., . . Davies, R. (2009). Never Waste a Good Crisis: A Review of Progress Since Rethinking Construction and Thoughts for Our Future. Loughborough University. Constructing Excellence. Retrieved from http://www.constructingexcellence.org.uk/

[8] HM Government. (2013). Construction 2025. Industrial Strategy: Government and Industry in Partnership.

[9] British Standards Institution. (2010). BS6079-1:2010: Project Management. Principles and Guidelines for the Management of Projects. British Standards Institution. Retrieved April 20, 2018, from https://www.shop.bsigroup.com

[10] Association for Project Management. (2012). APM Body of Knowledge. Buckinghamshire, England: Association for Project Management.

[11] Chartered Institute of Building. (2016). Code of Practice for Programme Management in the Built Environment. Chichester: Wiley Blackwell.

[12] Winch, G. M., \& Kelsey, J. (2005). What Do Construction Project Planners Do? International Journal of Project Management, 23(2), 141-149. https://doi.org/10.1016/j.jpproman.2004.06.002

[13] Laufer, A., \& Tucker, R. L., (1987). Is Construction Project Planning Really Doing Its Job? A Critical Examination of Focus, Role and Process. Construction Management and Economics, 5(3), pp. 243-266. https://doi.org/10.1080/01446198700000023

[14] Koskela, L. (1992). Application of the New Production Philosophy to Construction. 72. Stanford: Stanford University.

[15] Ballard, H. G. (2000). The Last Planner System of Production Control. Doctoral Dissertation. University of Birmingham. 